\section{CHRONIC RHEUMATIC ARTHRITIS.}

BY HUGH LANE, L.R.C.P. EDIN, M.R.C.S. SURGEON TO THE ROYAL MINERAL WATHR IIOSPITAL, AND HONORARY MEDICAI OFFICER TO THE ROYAL UNITED HOSPITAL, BITH.

Witur respect to the subject of rheumatic and rheumatoid arthritis, which I have frequently discussed in the columns of Trie LANCET, the following case may prove of interest as exemplifying the distinctions originally drawn by Mr. C. T. Griffiths and myself-viz., that one disease, rheumatic arthritis, is an affection of rheumatic origin, local in character, in which the neural element is absent; and the other, rheumatoid arthritis, is a general disease of debility, having no connexion with rheumatism, in which the neural element plays a conspicuous part, and which occurs in cases with strong hereditary histories of gout and struma or phthisis, and in which the last stage is osteo-arthritis.

The patient, a female aged forty-three (father living and healthy, aged seventy ; mother died of paralysis, aged sixtyfour; two brothers and six sisters all living and strong), was healthy and well till nineteen years of age, when she had subacute rheumatism (attributed to sleeping in a damp bed), which hardly confined her to bed, but she felt pain and stiffness in her knees, shoulders, elbows and thumb-joints. These joints never got better, but remained stiff and painful after the attack, she feeling every change in the weather. This is a strong rheumatic symptom, for in rheumatoid arthritis the weather does not affect the course of the illness to the same extent, and patients will frequently say they feel better in damp or cold weather. She had subsequent subacute attacks in which more joints suffered until every joint got affected, with the exception of the temporo-maxillary articulation, and she ultimately became quite bedridden and has remained so for twenty years. The catamenia came on at fourteen years, and continued regularly. No phthisis or gout in the family history. Every joint is firmly and rigidly ankylosed, not even the slight movement which can nearly always be obtained in the ankylosis occasioned by osteo-arthritis (the last stage of rheumatoid arthritis), in which case the ankylosis is caused by obstruction to movement by bony outorowths. The joints are stiffly fixed; no increase in the size of a single joint beyond an apparent enlargement due to wasting of the limbs, which now are so emaciated that they present the appearance of merely skin and bone. The only joint at present in which there is any movement is the jaw, and that is healthy. She some years ago, when not quite so helpless in the spine, used to take care of her sister's child, and alter its position by raising it with her teeth, but now she cannot even turn her head. The joints of the limbs nearly all contracted, elbows at right angles and close to side; knees also bent up, and fingers contracted in palm of hand; skin healthy, colour good; no great anxmia ; heart fairly normal, but slight mitral bruit; pulse 60 to minute; bowels opened only once a week, and then only with the aid of an enema or apcrient; general health good; eats fairly well, and seems cheerful, frequently singing to the children; and, strangely enough, she is plump in the face, the muscles of which do not a prear to be wasted; feels well, except in the springtime of the year ; urine, sp. gr. 1020; sugar absent; albumen absent; reaction acid; oxalates absent; small quantity of mucus; panses about $300 \mathrm{gr}$. of urea daily.

'The following interesting points show the difference between the two conditions chronic rheumatoid arthritis and chronic rheumatic arthritis ${ }^{1}:-1$. Family history reveals an utter absence of gout and phthisis, which I have always asserted are found in that of rheumatoid arthritis, 2. No nervous sumptoms, neuroses, formication, tingling of limbs, sweating, numbness, pigmentation of skin, and no constitutional syniptoms - simply a joint affection; and although every limb is wasted, there is no evidence of general organic progressive disease, as in rheumatoid arthritis, where the muscular atrophy is one of the earlier symptoms, and as a rule precedes the joint affection. 3. Character of joints affected: all firmly ankylosed, with no enlargement, but of course apparently so, owing to the wasting of the surrounding structures; no effusion, and there are absolute flexion and fixation in this case. Flexions of joints in the

I Differentiation in Rheumatic Diseases so called. By Hugh Lane. J. and A. Churchill. vast majority of cases are of rheumatic origin, while in rhen. matoid arthritis frequently the fingers are hyperextended, but this is not the rule. The character of the joints affected in rheumatic arthritis contrasts strongly with the condition of the joints in rheumatoid arthritis, where the joints are always swollen, with a sensation under the fingers, as if of tluctuation, the swelling commencing some distance above the joint and terminating some distance below it, and always of a more or less spindle-shape. 4. In this case the temporo-maxillary is the only articulation exempt, oddly enough bringing evidence to prove the correctness of my view, that this joint plays an important part in the differentiation between these diseases, ${ }^{2}$ where I pointed out that in rheumatoid arthritis the joints most used are the first to go ; and, as everyone must talk and eat, this joint never enjoys a lengthened period of repose, and therefore is frequently the seat of the disease, while in rheumatic arthritis it is rarely affected. 5. Her good spirits; not so in rheumatoid arthritis, when depression is an early and constant feature. 6. The symmetry of the joints affected is another point of distinction but that is not essential in this case, as nearly every joint in the body is affected. 7. No marked anæmia, which is so prominent a symptom all through rheumatoid arthritis. 8 Slight mitral bruit, but slow acting heart, soft pulse In
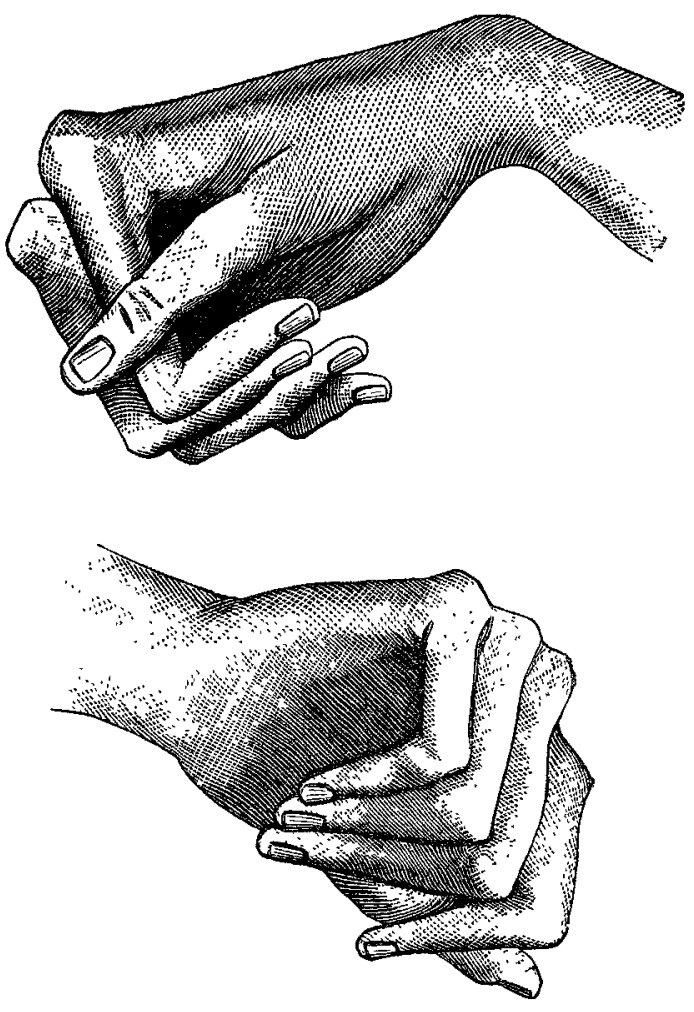

rheumatoid arthritis the heart's action is rapid, usually over 100 , no anæmic bruit is found and there is a hard pulse. 9. Although the illness in this case developed before the usual period, the preponderance of evidence points to this being case of rheumatic arthritis, as described by Mr. Griffiths and myself.

The importance of this proper differentiation cannot bo over-estimated, as upon this treatment entirely hinges-the treatment of rheumatism and rheumatic arthritis by antirheumatic medication and non-nitrogenous diet \&c.; gout and gouty arthritis by appropriate medicinal treatment, depletion, diet \&c. ; and, finally, that of rheumatoid arthritis, in which case the strumous element so much predominates ${ }^{3}$ that it has now caused quite a revolution in the treatment, approximating it to that of struma or phthisis, by cod-liver oil, tonics, stimulation and build. ing up. It must be clearly and distinctly regarded, not as a result of rheumatism or as a hybrid condition, the diagnosis and nomenclature of which were scamped under the absurd appellation of rheumatic gout, but as a distinc disease possessing strong and peculiar features of its own, an offspring of the hereditary tendencies of gout and scrofula. Of course, from the facts of a single case like this it is impossible to deduce any absolute principles, but I hope it will be a link, though a single link, in the chain of evidence which $I$ have previously brought forward in a large number of cases. Bath.

2 Vide ThE LANCET, Dec. 26th, 1891, and following numbers 3 Vide Differentiation in Rheumatic Diseases so-called. 